Continuing
medical
education

\section{Evaluation of behavioural interventions in HIV/STI prevention}

Judith M Stephenson

\section{Introduction}

Behavioural interventions in HIV/STI prevention are a subject of considerable interest and debate. In the absence of effective vaccines such interventions are key to HIV/STI control strategies, but the question of what actually works remains a challenging one. Published research aiming to evaluate the effectiveness of behavioural interventions has been reviewed and mostly found wanting. ${ }^{12}$ This article considers why the evaluation of behavioural interventions is often complex, and attempts to unravel some of the surrounding controversy. Questions include what is meant by behavioural interventions? how can they be evaluated? and what can they be expected to achieve in HIV/STI prevention?

\section{What is meant by behavioural intervention?}

Various terms, including behavioural, psychosocial, and lifestyle, are used to describe very different kinds of interventions designed to change a wide range of human behaviours. For example, the intervention may range from a brief exchange of information or advice to long term, intensive psychological "counselling" or therapy. And the aim may be to change the smoking, exercise, dietary, or sexual behaviour of individuals, small "high risk" groups, or whole communities. In the context of HIV/STI prevention, a behavioural intervention is one that seeks to reduce the risk of acquiring or passing on HIV or other STI by changing behaviours that lead to transmission of infection, principally sexual and injecting behaviours. This still encompasses a wide range of possibilities, as the link between behaviour change and transmission of infection may be fairly direct (for example, consistent use of condoms between known HIV discordant sexual partners) or much more indirect (for example, raising self esteem or negotiation skills among sexually inexperienced young people to reduce the likelihood of high risk sexual behaviour in the future).

Behavioural interventions have been based on a number of psychological models such as theories of reasoned action, ${ }^{3}$ self efficacy, ${ }^{4}$ and readiness to change. ${ }^{5}$ For example, the model of Prochaska and Diclemente ${ }^{5}$ asserts that a series of discrete changes to thoughts and actions are required before behaviour change can be achieved. Whatever the underlying psychological model, the development of a promising behavioural intervention requires careful exploratory research into the determinants of sexual behaviours and the cultural context, values, beliefs, and community norms of target groups. This is the role of qualitative and psychological research which is crucial to the design and implementation of promising interventions but beyond the scope of this brief article. The following focuses on how to evaluate the impact of behavioural interventions in HIV/STI prevention, with particular reference to the role of randomised trials.

\section{How can behavioural interventions be evaluated?}

Considerable debate still centres on whether the randomised controlled trial should be considered the gold standard for evaluating the impact of behavioural interventions. ${ }^{26-8}$ It has been claimed that "traditional experimental methods are often hopelessly inapplicable to studies of risk behaviour . . .and of limited feasibility in the evaluation of fledgling community public health programmes." " By contrast, the evidence based approach to the evaluation of healthcare interventions views the randomised trial as the optimal research design for this purpose because of its ability to minimise bias and avoid false conclusions about what works and what does not. To accept the randomised trial as the gold standard, however, is not to deny its limitations and challenges, particularly in the behavioural or psychosocial field. ${ }^{6}$

A frequent objection to randomised trials of behavioural interventions relates to the ethics of "withholding" the intervention from a control or comparison group. This is reminiscent of the debate that flourished when new therapies were increasingly subject to clinical trials in mainstream medicine. Now it is well accepted that anti-HIV therapies should be evaluated in clinical trials before being made available to all who might benefit. Whether it is a drug or a behavioural intervention, the ethical argument for randomised trials rests on "equipoise"- that is, genuine uncertainty that the intervention will actually result in more good than harm. A good example is school sex education, where there are few rigorous studies of the long term effects on the sexual health of young people, but entrenched views among professionals and the general public that school sex education has a positive, negative, or simply no effect on young people. ${ }^{10}$ Without well designed trials of sex education, uncertainty and dogma will frustrate efforts to seek a better way forward. Faced with the enormity of the HIV epidemic and the urgent need to find effective behavioural interventions to combat its spread, there has been a tendency to think that action (implementing behavioural interventions) must be preferable to inaction, but 
this is essentially a leap of faith rather than a scientific approach, and experience shows that well meaning measures may not work as intended. ${ }^{11}$

It has also been argued that randomised trials are not appropriate for evaluating behavioural interventions because they ignore the complexity of behavioural and psychosocial interventions. ${ }^{8}$ Behavioural trials clearly differ in this respect from clinical trials in which the intervention is a new drug whose dose and route of administration have already been established in phase I and II drug trials. By comparison, behavioural or psychosocial interventions are more likely to resemble a "black box", in which the "active ingredient" has not be identified at the outset. For example, if a support group based on cognitive behavioural therapy proves effective in reducing sexual risk behaviour, is it the cognitive behavioural component of the treatment that works, or is non-specific group support more important? To distinguish confidently between these two possibilities, each component would need to be tested in additional experiments, or in a single trial with multiple arms. Either option is often too costly or lengthy to be realistic. A decision must therefore be made at the outset whether or not to conduct a pragmatic trial, in which the whole intervention package is tested to see whether it advances current healthcare provision, regardless of identifying the active ingredient. Despite some clear differences between clinical and behavioural intervention trials, the ethical principle of establishing that an intervention does more harm than good is unaltered by its complexity. There are plenty of examples of complex chemotherapeutic regimens, for example, that are routinely subject to randomised trials. Perhaps the greatest challenge, in the behavioural field, is to design and conduct trials in such a way that the delivery of the intervention can be standardised as much as possible and the black box made more transparent, so that the benefits of behavioural interventions, once demonstrated in a controlled trial, can be replicated in real life.

\section{What can behavioural interventions achieve in HIVISTI prevention?}

A key issue here is the choice of outcomes by which to assess the success of a behavioural intervention. ${ }^{12}$ The ultimate goal of such interventions is to reduce the rate of new HIV/STI infections in defined groups or populations, and many innovative interventions have been set up throughout the world with this goal in mind, but no randomised trial has yet reported the impact of a behavioural intervention on HIV incidence. ${ }^{213}$ This partly reflects the fact that HIV incidence is relatively low, even in high risk groups (for example, around $5 \%$ per year in HIV discordant heterosexual couples, ${ }^{14}$ or $4 \%$ in homosexual men attending central London genitourinary medicine clinics $^{15}$ ). Large, costly trials are needed to reliably detect an epidemiologically or clinically meaningful difference (between intervention and control groups) in an uncommon endpoint like incident HIV infection.
Acute (non-HIV) STIs are suitable endpoints for behavioural intervention trials because they are important causes of morbidity in themselves, they occur more commonly than HIV infection, and have been clearly shown to increase the risk of HIV transmission. ${ }^{16}{ }^{17}$ In the context of a controlled trial, care needs to be taken that the opportunity for diagnosis of STI is the same in intervention and control groups, otherwise any difference at the end of the trial may simply reflect greater efforts to diagnose STIs in one group than another.

Should studies rely on self reports of high risk sexual behaviour-for example, unprotected intercourse, as outcomes by which to assess the impact of behavioural interventions? Much has been written about the validity of self reported sexual behaviour which cannot be observed or verified directly, and the potential for social desirability bias. ${ }^{18}$ In a controlled evaluation trial, it is important to be aware that the way in which informed consent is obtained can increase the potential for such bias, particularly where "blinding" to the intervention of interest is not possible. This emphasises the importance of conveying equipoise (see above) to potential participants at the outset, so as to minimise inaccurate reporting of the desired outcome equally across comparison groups. The problem of social desirability bias can also be addressed by new data collection methods. Recent studies in men and women comparing computer assisted self interview (CASI) with conventional pen and paper interviewer questionnaires (PAPI) have shown that disclosure of sensitive or stigmatised behaviours, such as sex with prostitutes, is more likely to occur with CASI. ${ }^{19}$ Despite these methodological advances in improving the quality of self reported behavioural data, it seems wise to include objective outcomes of high risk sexual behaviour, such as STI markers, wherever possible.

A criticism frequently levelled at behavioural intervention trials is that they do not reflect the situation in real life. ${ }^{80}$ Rarely, however, do interventions of any sort have such a dramatic impact that their usefulness can be established outside a controlled research environment. Despite this, pragmatic trials can be designed to mimic real life conditions as closely as possible without compromising the scientific need to establish a link between intervention and specified outcome(s). Another issue closely related to "real life relevance" is the generalisability of behavioural interventions. Much is rightly made of the need to develop interventions that are culturally sensitive and appropriate to defined target groups. Features of a promising intervention for commercial sex workers in Bombay will obviously differ from those of an intervention aimed at homosexual men in London. But if an intervention is shown to be effective in a particular group, such as homosexual men in clubs and bars in the mid western United States, ${ }^{21}$ or school students in Uganda, how far can the findings be generalised to others? The answer depends on judgment rather than statistics, so the question of generalisability should be considered early 
on before the intervention is subject to evaluation. To evaluate an intervention that cannot be generalised beyond the setting in which it was conducted is clearly wasteful and ultimately unethical if the benefit of research experience cannot be passed on to others.

The setting in which behavioural interventions are evaluated can also affect the success of the study. In the United States, large, expensive, community randomised trials of lifestyle interventions to reduce cardiovascular disease (smoking and dietary fat reduction, exercise promotion, etc) have shown disappointingly small differences between intervention and control communities when conducted against a background of successful health promotion in the general population. ${ }^{20}$ By analogy, the success of a behavioural intervention trial may be influenced by rapidly changing social and community norms in relation to sexual behaviour. Psychological models that recognise this (for example, Prochaska and Diclemente ${ }^{5}$ ) may be especially relevant when considering the timing of intervention. Changing sexual behaviour through specific interventions is no easy task, and experience in other fields suggests that we should not expect large effects, as experimental interventions encompass only a segment of what social movement brings to bear. ${ }^{20}$ However, compared with other study designs, randomised trials are uniquely able to disentangle the effect of background influences on health related behaviour from those of an applied intervention. More large trials with objective outcome measures are needed to show just how much we can expect behavioural interventions to achieve in HIV/STI prevention.

\section{Summary}

There is an urgent need for well designed randomised trials to assess the impact of behavioural interventions at both individual and community levels in developed and developing countries. The relative lack of such studies partly reflects the particular challenges of applying randomised trials in this area. Although there are obvious differences between clinical and behavioural interventions, the principles underlying successful evaluation are not fundamentally different. Experience gained from clinical trial methodology over the past two decades should be applied and further developed to tackle the demands and challenges of evaluating behavioural interventions in HIV/STI prevention.

1 Oakley A, Fullerton D, Holland J. Behavioural interventions for HIV/AIDS prevention. AIDS 1995;9:479-86.

2 Kegeles S, Hart G. Recent HIV prevention interventions for gay men: individual, small-group and community-based studies. AIDS 1998;12:S209-15.

3 Fishbein M, Ajzen I. Belief, attitude, intention, and behavior: an introduction to theory and research. Reading, MA Addison-Wesley, 1975.

4 Bandura A. Social foundations of thought and action: a social cognitive theory. Engelwood Cliffs, NJ: Prentice-Hall, 1986.

5 Prochaska JO, Diclemente CC. Towards a comprehensive model of change. In: Miller WR, Heather N, eds. Treating model of change. In: Miller WR, Heather N, eds. Treating 1986.

6 Stephenson J, Imrie J. Why do we need randomised controlled trials to assess behavioural interventions? $B M F$ 1998:316:611-13.

7 Susser M. Some principles in study design for preventing HIV transmission: rigor or reality. Am f Public Health 1996; 86:1713-16.

8 Coates TJ, Aggleton P, Gutzwiller F, et al. HIV prevention in developed countries. Lancet 1996;348:1143-8.

9 Watters JK. Behavioural science in the AIDS epidemic. Lancet 1994;344:1312-13.

10 Dean M. Muddle over sex education. Lancet 1994;343: 1149.

11 Zenilman JM, Erickson B, Fox R, et al. Effect of HIV posttest counselling on STD incidence. $7 A M A$ 1992;267:843-

12 Aral SO, Peterman TA. Measuring outcomes of behavioral interventions for STD/HIV prevention. Int $\mathcal{F}$ STD AIDS 1996;7:30-8

13 Hayes R, Wawer M, Whiteworth J, et al for the HIV/STD Trials Workshop Group. Randomised trials of STD treatment for HIV prevention: report of an international workshop. Genitourin Med 1997;73:432-43.

14 De Vincenzi I, for the European Study Group on Heterosexual Transmission of HIV. A longitudinal study of human immunodeficiency virus transmission by heterosexual partners. N Engl f Med 1994;331:341-6.

15 Miller E, Waight PA, Tedder RS, et al. Incidence of HIV infection in homosexual men in London, 1988-1994. BMF infection in hom

16 Grosskurth H, Mosha F, Todd J, et al. Impact of improved treatment of sexually transmitted diseases on HIV infection in rural Tanzania: randomized controlled trial. Lancet 1995;346:530-6.

17 Cohen MS. Sexually transmitted diseases enhance HIV transmission: no longer a hypothesis. Lancet 1998; 351(Suppl III):5-7.

18 Catania JA, Gibson DR, Chitwood DD, et al. Methodological problems in AIDS behavioral research: influences on measurement error and participation bias in studies of sexual behavior. Psychol Bull 1990;108:339-62.

19 Turner CF, Ku L, Rogers SM, et al. Adolescent sexual behaviour, drug use and violence: increased reporting with computer survey technology. Science 1998;280:867-73.

20 Susser M. Editorial: The tribulations of trials - intervention in communities. Am f Public Health 1995;85:156-9.

21 Kelly JA, St Lawrence JS, Stevenson LY, et al. Community HIV/AIDS risk reduction. The effects of endorsements by popular people in three cities. Am F Public Health 1991;82 1483-9. 